\title{
Editorial
}

\section{Severe Acute Respiratory Syndrome: Preparedness, Management, and Impact}

\author{
Mark B. Loeb, MD, MSc
}

Approximately 21 months have passed since the World Health Organization officially confirmed the severe acute respiratory syndrome (SARS) outbreak. Originating in the southern Chinese province of Guandong, from a putative animal source, the transmission of SARS from an infected physician to other guests at the Hotel Metropol in Hong Kong led to global spread. Eight thousand ninety-six individuals were infected and 774 died. ${ }^{1}$ Characterizations of the etiologic agent ${ }^{2}$ along with initial clinical descriptions of the syndrome $\mathrm{e}^{3,4}$ were followed by reports of outcomes of affected patients. ${ }^{5}$ As the outbreak progressed, it became clear that this was largely a healthcare facility-based outbreak, with healthcare workers, patients, and visitors being at risk. The bulk of the evidence, namely the descriptive epidemiology and related observational studies, suggests that droplet spread is the most common form of transmission. ${ }^{6,7}$ Although airborne transmission has been proposed as a mode of spread, the extent to which this occurred remains controversial. 8

Why did SARS not become more widespread in the community? The answer lies in both the characteristics of the SARS coronavirus and the efforts made in the hospital to reduce spread. The SARS coronavirus is not highly transmissible. The relatively low reproductive number (the number of secondary cases generated by an infectious case) contributed to the lack of community-wide spread. ${ }^{9}$ The fact that the viral load was low initially then increased (rather than being high initially then decreasing, like other respiratory viruses) may have played a role. ${ }^{10}$ Because the transmissibility of the virus was highest after 5 days of symptoms, isolation of new cases acquired in the hospital was possible with diminished spread. There is empiric evidence to substantiate this, an increase in secondary cases of SARS when time to isolation with respect to onset of symptoms lasts beyond 5 days. ${ }^{1}$ Nosocomial spread was also characterized by superspreading events. Although devastating to individuals in hospitals, superspreading where there is high variance in the number of secondary cases that arise, somewhat counterintuitively, is not an efficient strategy for viral epidemic spread. ${ }^{9}$ Efforts to reduce spread in the hospital undoubtedly prevented transmission and saved lives. It has been estimated that delaying the institution of control measures by 1 week would have nearly tripled the size of the epidemic and would have increased the expected duration of the epidemic by 4 weeks. ${ }^{11}$ In contrast, the role of mass quarantine in the community has been more controversial, particularly given that it is likely that the numbers quarantined in many jurisdictions were excessive. ${ }^{12}$ However, at the time of the outbreak, decisions made necessarily needed to be conservative given the incomplete knowledge about aspects of the transmission of SARS, such as the role played by asymptomatically infected individuals.

A hard lesson learned from SARS was our lack of preparedness for emerging infectious diseases. In Canada, this was evident through failure to achieve a coordinated response at municipal, provincial, and national levels. This led to the creation of a new national public health agency. Given that hospital transmission was such a key characteristic of the spread of SARS, an important lesson for hospital infection control has been the artificial division between "public health" and "infection control"-infection control is "hospital public health." In this issue of Infection Control and Hospital Epidemiology, preparedness, management, and the impact of SARS are addressed in three articles.

In the first of the three articles, Srinivasan et al. 
describe the foundation for the Centers for Disease Control and Prevention's SARS preparedness and response plan for healthcare facilities. ${ }^{13}$ The article provides a concise summary of a response plan. The recommendations are clear and are accompanied by a concise rationale. Of particular appeal is that the recommendations are not prescriptive, but rather are a "guidance" where there is an acknowledgment of the existing gaps in knowledge. Key themes include the importance of unrecognized cases as a source of transmission, the role of respiratory hygiene and cough etiquette, and surveillance of high-risk groups. In Toronto, problems with case ascertainment persisted well into the outbreak, with clinical judgment failing to invoke SARS as the diagnosis of an elderly patient with a clinical presentation typical of postoperative pneumonia. Unfortunately, rapid laboratory tests with high diagnostic accuracy remain elusive. Reports of clinical prediction rules offer some optimism, but lack of prospective validation in a separate sample and poor specificity are limiting factors. ${ }^{14}$ Particularly given the difficulties in early diagnosis, the promotion of a respiratory hygiene and cough etiquette policy proposed by the Centers for Disease Control and Prevention makes sense. Although there is no direct evidence for efficacy, the policy has face validity, borrowing from the pediatric experience to reduce spread of respiratory syncytial virus. As is usually the case in infection control, surveillance is a key component. Healthcare workers, visitors, and patients are high-risk groups, and the degree and type of surveillance is dictated by factors such as global or local epidemiology or the presence or absence of transmission in the healthcare facility.

One of the unresolved issues in the management of SARS, as stated in the article by Srinivasan et al., is the designation of dedicated SARS hospitals. The article by Fung et al. describes the creation of a temporary isolation ward for SARS patients in Taiwan..$^{15}$ Although the title of this article mentions a "ward," this was in fact a satellite building of the Sung-Shan Military Hospital with four floors. To build this SARS unit, an exhaust fan was installed above the window in each private room on the second, third, and fourth floors. The first floor was divided into zones: clean for changing into and out of street clothes, intermediate for removing the inner layer of personal protective equipment, and a contaminated area for entering isolation rooms. Of the 196 patients with suspected SARS admitted to this unit, 34 (17\%) had SARS. Only one nurse developed laboratory evidence of SARS and this was attributed to a break in infection control practice. Although this article would support development of such a unit, SARS patients with greater severity of illness remained on the unit for a limited period compared with stable SARS patients (mean of 3.6 vs 9.4 days). Given that in the non-outbreak situation it is impossible to predict exactly when a patient with SARS (or another emerging respiratory pathogen) will present to the emergency department, all hospitals need to be prepared for this possibility. The situation reported by Fung et al. is atypical because this was not an existing functional hospital in the first place. It would make less sense to transfer patients with SARS from one hospital to another that is in active use because the latter is declared the dedicated SARS hospital.

Although there have been numerous studies of the various effects of SARS on healthcare workers, few studies have assessed psychological distress at the community level. The article by Leung et al. describes a telephone survey about SARS conducted among residents of Hong Kong and Singapore. ${ }^{16}$ The surveys, conducted toward the end of the SARS outbreak, allowed the comparison between Hong Kong, where a more dramatic outbreak occurred, and Singapore. The anxiety levels, as well as nonspecific symptoms, were significantly higher in Hong Kong than in Singapore, raising the possibility of a higher incidence of psychosomatic illness. Although some of the behaviors could be clearly categorized as adaptive, such as increased compliance with respiratory etiquette in Hong Kong compared with Singapore, other responses, such as face mask wear in the community of $79 \%$ in Hong Kong versus $4 \%$ in Singapore, were more questionable. Perhaps some of the increased anxiety and use of face masks was due to the large superspreading events in Hong Kong, such as at Amoy Gardens. Further research, using well-defined theoretical constructs, is needed to understand the determinants of adherence to adaptive behaviors in large outbreaks.

Can the lessons learned from SARS help us with avian influenza? Fortunately, there have been no reports of efficient human-to-human transmission of H5N1. Nevertheless, a case-fatality rate of approximately $75 \%$ of cases in Vietnam and Thailand is ominous. The hospital preparedness plan for SARS described in this issue of Infection Control and Hospital Epidemiology would help for imported cases of the current H5N1 influenza strain. ${ }^{13}$ Surveillance for febrile respiratory syndromes would help with the early identification and isolation of cases. A strain of avian influenza that can be efficiently transmitted from person to person would, of course, pose a threat of an entirely different magnitude, as it is unlikely that initial search-and-contain efforts would be successful in stopping the virus from spreading widely. As with SARS, infection control efforts would need to focus on reducing the risk of transmission in the hospital through ensuring use of proper isolation precautions and promoting hand hygiene. Additional efforts would include, but would not be limited to, assisting with the delivery of vaccine and antiviral agents to hospital personnel, maintaining up-todate line lists of immunized and nonimmunized staff (if vaccine is available), developing strategies for diagnostic testing, and participating in regional pandemic teams. In such circumstances, maintaining effective lines of communication from the hospital to regional and national agencies would be key. Many of the strategies described by Srinivasan et al. in the Centers for Disease Control and Prevention preparedness plan for SARS would apply, such as coordinated surveillance efforts with local and state health departments, promotion of respiratory etiquette, restricting visitor access, healthcare worker and patient surveillance, and staff education. ${ }^{13}$ The concept of having dedi- 
cated assessment facilities for individuals requiring nonurgent care outside of acute care hospitals would apply. For individuals requiring urgent care, the concept of rapid creation of hospital wards where non-critically ill patients could be managed deserves consideration, similar to the proposal by Fung et al., although isolation is droplet. ${ }^{15}$ The findings by Leung et al. are also relevant as it is likely that the psychological impact of a pandemic would be far greater than that of SARS, particularly if the strain of influenza is virulent and has a predilection for younger individuals. ${ }^{16}$

SARS may or may not re-emerge. Regardless, the insight that we have gained into healthcare facility responsiveness should serve us well as we prepare to deal with a far greater challenge, that of pandemic influenza.

\section{REFERENCES}

1. World Health Organization. Cumulative Number of Reported Probable Cases of Severe Acute Respiratory Syndrome (SARS). Geneva: World Health Organization; 2004.

2. Drosten C, Gunther S, Preiser W, et al. Identification of a novel coronavirus in patients with severe acute respiratory syndrome. $N$ Engl J Med 2003;348:1967-1976

3. Poutanen SM, Low DE, Henry B, et al. Identification of severe acute respiratory syndrome in Canada. N Engl J Med 2003;348:1995-2005.

4. Lee N, Hui D, Wu A, et al. A major outbreak of severe acute respiratory syndrome in Hong Kong. N Engl J Med 2003;348:1986-1994.

5. Booth CM, Matukas LM, Tomlinson GA, et al. Clinical features and short-term outcomes of 144 patients with SARS in the greater Toronto area. JAMA 2003;289:2801-2809.

6. Varia M, Wilson S, Sarwal S, et al. Investigation of a nosocomial outbreak of severe acute respiratory syndrome (SARS) in Toronto, Canada. CMAJ 2003;169:285-292.

7. Seto WH, Tsang D, Yung RW, et al. Effectiveness of precautions against droplets and contact in prevention of nosocomial transmission of severe acute respiratory syndrome (SARS). Lancet 2003;361:1519-1520.

8. Yu ITS, Li Y, Wong TW, et al. Evidence of airborne transmission of the severe acute respiratory syndrome virus. $N$ Engl J Med 2004;350:17311739.

9. Lipsitch M, Cohen T, Cooper B, et al. Transmission dynamics and control of severe acute respiratory syndrome. Science 2003;300:1966-1970.

10. Peiris JS, Chu CM, Cheng VC, et al. Clinical progression and viral load in a community outbreak of coronavirus-associated SARS pneumonia: a prospective study. Lancet 2003;361:1767-1772.

11. Wallinga J, Teunis P. Different epidemic curves for severe acute respiratory syndrome reveal similar impacts of control measures. $\mathrm{Am} \mathrm{J}$ Epidemiol 2004;160:509-516.

12. Centers for Disease Control and Prevention. Efficiency of quarantine during an epidemic of severe acute respiratory syndrome: Beijing, China, 2003. MMWR 2003;52:1037-1040.

13. Srinivasan A, MCDonald LC, Jernigan D, et al. Foundations of the severe acute respiratory syndrome preparedness and response plan for healthcare facilities. Infect Control Hosp Epidemiol 2004;25:1020-1025.

14. Leung $\mathrm{G}$. A clinical prediction rule for diagnosing severe acute respiratory syndrome in the emergency department. Ann Intern Med 2004;141: 333-342.

15. Fung C-P, Hsieh T-L, Tan K-H, et al. Rapid creation of a temporary isolation ward for patients with severe acute respiratory syndrome in Taiwan. Infect Control Hosp Epidemiol 2004;25:1026-1032.

16. Leung GM, Quah S, Ho L-M, et al. A tale of two cities: community and psychobehaviorial surveillance and related impact on outbreak control in Hong Kong and Singapore during the severe acute respiratory syndrome epidemic. Infect Control Hosp Epidemiol 2004;25:1033-1041.

\section{Medical News}

Edited by Gina Pugliese, RN, MS; Martin S. Favero, PhD

\section{Centers for Medicare \& Medicaid Services Surgical Infection Prevention Project Expands to Include Other Surgical Complications}

The Centers for Medicare \& Medicaid Services (CMS) has expanded its Surgical Infection Prevention Project (SIPP) to include other surgical complications. This expanded initiative, called the Surgical Care Improvement Project (SCIP), is a national partnership of organizations committed to improving the safety of surgical care through the reduction of postoperative complications. The goal is to reduce surgical complications nationally by $25 \%$ by the year 2010 in four target areas: surgical-site infections and cardiac, respiratory, and venous thromboembolic complications.

In addition to the proper use of antibiotics to prevent surgical-site infections, examples of other measures that are being evaluated include preventing hypothermia during the procedure, maintaining high levels of inspired oxygen, controlling serum glucose within certain limits, and avoiding shaving of the operative site.
A SCIP steering committee of public and private organizations, including the Society for Healthcare Epidemiology of America, has been working since 2003 to develop a quality improvement framework to improve both patient safety and the quality of care for surgical services nationwide. In preparation for an official launch in the summer of 2005, several developmental activities are currently under way, including completion of a three-state demonstration pilot, formation of four technical expert panels to provide specialized guidance for improving each of the four target areas, and development of information, materials, and evidence-based strategies to help hospitals and their professional staffs participate-and succeed-in this national effort. More information is available at www.MedQIC.org/scip. 